\title{
Contratación y capacitación: Factores de permanencia del capital humano en las PYMES
}

\section{Recruitment and training: permanence factors of human capital in PYMES}

\author{
Noemi Vega Lugo ${ }^{a}$, Brenda Hurtado Vega ${ }^{b}$, José Sergio Rodríguez Martínez ${ }^{c}$
}

\begin{abstract}
:
Being SMEs in Mexico one of the key sectors to boost the country's economy, it is necessary that they be endowed with tools that allow them to boost the human capital they possess and understand that their size does not limit them to establish essential administrative aspects not only for its proper operation but to increase its productivity.

Thus, recruitment, selection, hiring, induction and training, among other no less relevant issues, should be approached from a simple but enriching perspective, which allows these small and medium-sized companies to start a series of exercises that contribute to their positioning in the market. market and why not, position itself as one of the top competitors not only nationally but also internationally
\end{abstract}

Keywords:

Business, PYMES, human capital, hiring, training

Resumen:

Siendo las PYMES en México uno de los sectores claves para impulsar la economía del país, es necesario que se les dote de herramientas que les permitan impulsar el capital humano que poseen y comprender que su tamaño no les limita a establecer aspectos administrativos indispensables no solo para su adecuado funcionamiento sino para aumentar su productividad.

Es así como el reclutamiento, la selección, contratación, inducción y capacitación, entre otros temas no menos relevantes deben abordarse desde una perspectiva sencilla pero enriquecedora, que permita a esas pequeñas y medianas empresas iniciar una serie de ejercicios que coadyuven a su posicionamiento en el mercado y porque no, situarse como uno de los altos competidores no solo a nivel nacional sino internacional.

\section{Palabras Clave:}

Empresa, PYMES, capital humano, contratación, capacitación

\section{Introducción}

Mucho se habla del impulso que gobiernos federales, estatales y municipales dan a las Pequeñas y Medianas Empresas (PYMES) en México, pues estas de acuerdo con datos emitidos por la Secretaría de Economía representan el $99.8 \%$ y generan el $73.8 \%$ del empleo. [1] Sin embargo es un hecho la dificultad a la que se enfrentan por atribuir a su tamaño la limitante para establecer estrategias y controles administrativos que les permitan trabajar de manera sólida para ser competitivos en un mercado para muchas de ellas altamente difícil. Es

\footnotetext{
a Universidad Autónoma del Estado de Hidalgo, Programa Educativo de Turismo, https://orcid.org/0000-0002-6544-2446, Email: noemvl@uaeh.edu.mx

b Universidad Autónoma del Estado de Hidalgo, Dirección de Superación Académica, https://orcid.org/0000-0002-9164-7330, Email: brenda_hurtado@uaeh.edu.mx

c Universidad Autónoma del Estado de Hidalgo, Programa Educativo de Turismo, https://orcid.org/0000-0001-5064-8022, Email: sergior@uaeh.edu.mx
} 
importante que las Pymes inicien con una estructura administrativa formal independientemente de que muchas de ellas sean consideradas como familiares, la base administrativa permitirá un desarrollo adecuado no solo en su infraestructura sino en su activo más valioso representado por el Capital Humano.

Es así como la contratación y la capacitación se convierten en parte medular para su buen funcionamiento, aspectos que son abordados de manera sencilla y clara en el Libro Contratación y capacitación: Factores de permanencia del capital humano en las PYMES, cuyos coordinadores Tirso Javier Hernández Gracia, Karina Valencia Sandoval, Danae Duana Ávila y Ma. Del Rosario García Velázquez en trabajo conjunto con Profesores Investigadores y alumnos del Colegio de Postgraduados (Campus Montecillo), la Universidad Autónoma de Tlaxcala y la Universidad Autónoma del Estado de Hidalgo presentan al lector en once capítulos un panorama amplio e interesante de la importancia de la contratación y capacitación en las PYMES mexicanas. [2]

En su primer capítulo denominado "La contratación de Personas" se describe la fundamentación legal y la importancia de generar un contrato de trabajo que de acuerdo a la Ley Federal del Trabajo en su artículo 20 y 21 lo define como "en virtud del cual una persona se obliga a prestar a otra un trabajo personal subordinado, mediante el pago de un salario", además de presentar la clasificación de los mismos y resaltar el hecho de que su inexistencia no priva al trabajador de sus derechos y al patrón de sus obligaciones. Cerrando en la última parte de este capítulo con ocho acciones a considerar previas a la celebración de la contratación de un trabajador.

En un segundo capítulo de esta obra titulado "Herramientas para la contratación de personal" se aborda el tema de la Psicología Organizacional o Psicología del Trabajo que de acuerdo a Frank Landy y Jeffrey Comte $(2005)^{1}$ puede definirse como "la aplicación de principios, teorías e investigaciones psicológicas en organizaciones como corporaciones, empresas, organizaciones gubernamentales, organizamos públicos, gremiales, académicas, comunitarias y de salud". [3]

En lo particular de este capítulo los autores se centran en las pruebas psicométricas como herramienta para la contratación de personal, describiendo las características que éstas deben poseer como confiabilidad y validez; su clasificación e indicadores que miden.

Un tercer capítulo nos lleva a un tema por demás complicado para cierto número de personas que laboran en una empresa y es el denominado "Discriminación en el proceso de selección".
De acuerdo al Consejo Nacional para prevenir la discriminación (CONAPRED, 2021):

"La discriminación es una práctica cotidiana que consiste en dar un trato desfavorable o de desprecio inmerecido a determinada persona o grupo, a veces nos percibimos, pero que en algún momento la hemos causado o recibido". [4]

Entre algunos de los motivos por los cuales se puede dar la discriminación se encuentran las características físicas, forma de vida, preferencias sexuales, edad, origen étnico, discapacidad, embarazo, condición social o económica, entre otras y que sueles presentarse dentro y fuera del ámbito laboral.

Lamentablemente algunas empresas consideran estos puntos como parte de los requisitos al contratar personal, donde su inserción a la empresa no depende de su capacidad sino de una discriminación abierta o disfrazada en algunos centros de trabajo y que los lleva a negarse la oportunidad de contar con personal altamente capaz y competitivo.

Los autores de este capítulo llevan al lector a visualizar la legislación existente en México referente a este tema, así como a identificar la discriminación de acuerdo al género para finalizar con la presentación del Modelo de Equidad de Género emitido por el Banco Mundial.

Dentro del cuarto capítulo titulado "La necesidad de capacitación dentro de las PYMES" se describe de manera clara el concepto de capacitación, beneficios e importancia dentro de estas empresas, así como la necesidad de considerar a la capacitación y desarrollo como una de las partes medulares para hacer frente a un mundo cada vez más competitivo y a últimas fechas colapsado por situaciones fuera de control ocasionadas por la pandemia originada por el COVID-19 y que seguramente involucra un cambio de paradigma que recae en la forma de ofrecer un producto o servicio y en donde la capacitación no podrá estar aislada.

El capítulo cinco bajo el título "Legislación en el marco de la capacitación" refiere de manera clara los antecedentes de la capacitación y su reglamentación en México para lo cual citan lo dicho en la Constitución Política de los Estados Unidos Mexicanos, La ley Federal del Trabajo, Ley Orgánica de la Administración Pública, Reglamento Interior de la secretaria del Trabajo y Previsión Social.

Pasando a un sexto capítulo denominado "Estrategias de capacitación" se proporciona un panorama general de las estrategias de capacitación más importantes a nivel nacional e internacional entre ellas la metodología 
presencial en sus modalidades de seminario, curso, taller, especialización, diplomado, aprender haciendo, modelo de casos entre otros; Programa de Formación Acción, Coaching Ejecutivo; E-learning; Webinarios; Capacitación por competencias laborales; Entrenamiento y educación vocacional, así como Aprendizaje informal, describiendo cada una de ellas y presentando al lector un amplio panorama de cual o cuales estrategias podría elegir para la formación y desarrollo de su talento humano.

En el capítulo ocho titulados "Outsourcing como estrategia de contratación y capacitación" se proporciona al lector información sobre algunos conceptos de este tema tan utilizado y controversial en México, sobre todo por las condiciones en que son atendidas las necesidades de los trabajadores de las empresas que proporcionan este servicio.

Partiendo de la descripción de sus antecedentes, un panorama mundial y su aplicación en México lleva al lector a abordar esta herramienta desde varias perspectivas ampliando el escenario para que pueda tomar decisiones al respecto.

Un noveno capítulo denominado "La comunicación como un proceso fundamental en la contratación y capacitación sitúa al lector en la comprensión y análisis de la importancia que tiene ésta dentro de una empresa independientemente de su tamaño y giro, describe a la comunicación como la parte medular de cualquier organización, define los flujos de información, estrategias en el proceso de contratación de personal y en el proceso de capacitación, hasta llegar a la gestión de la comunicación interna.

En los dos últimos capítulos titulados "La capacitación y la ejecución de PYMES, para la comercialización: una historia de éxito" y "La capacitación como factor de desarrollo empresarial, el caso de la sociedad cooperativa Cédula Virreynal De 1577" se presentan dos casos de estudio donde claramente se visualiza la importancia y el desarrollo que representa el abordar los temas contemplados en los capítulos previos.

A manera de conclusión se puede afirmar que esta obra deja ver de manera clara y precisa la importancia de contar con adecuadas herramientas que permitan contratar a personal capaz para cubrir una vacante y que para quienes ya llevan tiempo prestando sus servicios resulta primordial no solo el estar capacitados, sino que se pueda lograr realmente un desarrollo. [5]

Los diversos temas abordados por estos autores reflejan el compromiso de las PYMES por el cuidado de su capital humano y les permite comprender que de ellos depende la productividad de la empresa y el mantenerse competitiva dentro del mercado.

Esta obra representa una alternativa ideal para los estudiosos en asignaturas de Administración, Administración de Recursos Humanos o Personal, así como para los de Psicología, que de manera breve y clara describen los temas de la contratación y capacitación desde varios puntos de vista logrando así su comprensión integral.

\section{Referencias}

[1] Economía, S. d. (24 de Febrero de 2020). Secretaria de Economía Obtenido de Reporte T-Mec: https://www.gob.mx/cms/uploads/attachment/file/536695/ReporteTMEC_n34-esp_20200224_.pdf

[2] Hernández Gracia, T., Valencia Sandoval , K., Duana Ávila, D., \& García Velázquez, M. (2016). Contratación y capacitación: Fectores de permanencia del capital humano en las PYMES. México: Plaza y Valdes.

[3] Anaya, F. (2018). Ley Federal del Trabajo comentada. Primera ed. México: Porrúa. pp. 176- 177.

[4] (CONAPRED), C. N. (25 de Junio de 2021). Secretaria de Gobernación. Obtenido de CONAPRED: https://www.conapred.org.mx/index.php?contenido=pagina\&id=84\&id _opcion $=142 \&$ op $=142$.

[5] Landy, F., \& Conte, J. Psicología Industrial. México: McGraw-Hill Interamericana; 2005: 20-30. 